\title{
Study of Facial Expression Analysis of Feature Extraction Based on Lip Movement for User Authentication
}

\author{
Dolly Shah \\ Department of CSE, SVITS, \\ Indore (M.P), India
}

\author{
Dinesh Chandra Jain \\ Department of CSE, SVITS, \\ Indore (M.P), India
}

\begin{abstract}
We are living in the 21st century, As the technology grows the security issues are gradually increasing and for those issues there is always a need for improvements in existing methods or there is a hope for new ideas towards this field. As we use the technology from everywhere in over daily life there is a challenge comes regarding data security or information hiding from the outer world and for this the human being is always helping us to words this problem. The human body is always a hot topic for the researchers for their research here we are using the same thing for over research purpose. The DNA analysis, Eye and finger print analysis be widely use for the security issues with this series we are using the Lips for over research. As a contribution we are proposing a Lip based security system for this we need to use the combination of several independent methods like facial expression analysis, talk analysis and text analysis by using these things we will be provide a new authentication idea for the world towards data security or system authentication.
\end{abstract}

\section{General Terms}

Facial Movements, Message Authentication Codes, Lip Based Security, Talk Analysis, Integrity.

\section{Keywords}

Facial Expression, Security, System Authentication

\section{INTRODUCTION}

In recent years, computer system and software protection have significant importance in the field of software engineering. The significance about software protection has been an eye-catching area of research as software itself is vulnerable to theft and misuse. Because of various software threats and attacks, a number of software security schemes have be developed by various researches in the literature. Previous protection approaches were largely partial to direct media-based protection and serial numbers. Various approaches such as processor dependent code, encryption, and obfuscation have been developed for the software protection.

As a security concerned, cryptographic approaches have received the greatest academic attention, because of its classic mathematical data-manipulation algorithms involving secret keys, encryption algorithms for confidentiality and Message Authentication Codes (MACs) and digital signature algorithms for real-time authentication, data origin authentication, integrity or non-repudiation[1].

To words the information security system the Digital image based process system additionally compete a good role during this field. Face reorganization or gesture based authentication systems exist for the system security. The necessity for AN automatic lip-reading system is ever increasing. Infect, today, extraction and reliable analysis of facial movements frame a very important part in several multimedia system systems such as videoconferencing, low communication systems, lip-reading systems [2].

One primary reason is that the lip shape is elastic, and hence it is difficult to model it by a rigid model. Moreover, the low contrast between the lip and the neighboring face regions and the complex illumination variations around it impede the achievement of accurate extraction results [3].Indeed, the same way humans combine audio-visual stimuli to recognize speech, the complementary information extracted from lip motion is expected to improve the recognition results when significant environmental noise is present in the audio channel. Furthermore, recent works on visual speech synthesis showed that video-realistic artificial synthesis of talking faces has good intelligibility results [4]

The human information, victimization these algorithms researchers have developed new applications like Traffic movement following, finding out plant root growth, Landing modules of rovers, Hand posture analysis, Human posture analysis, Gesture controlled gaming, Lip movement for user authentication, picture taking, Robotic surgical operation, breathing motion estimation[5].

Problems in lip shape tracking are further compounded by variations in lip colour, lighting and skin colour across different individuals. There exist a number of different methods for lip tracking [6].Traditionally lip options have been utilized in speech recognition, but of late they need also been found useful as a biometric symbol. Since this application of lip options is comparatively recent, several limitations may be ascertained. Firstly, majority of the research has targeted on victimization physical attributes of the lips, like shape and look [7].

\section{LITERATURE SERVEY}

In this section we analyze the past and present of the issues which are related to our area.

According to Yiu-ming Cheung work they need introduced the framework of LCACM, through which the colour objects incorporating complex appearances or intensity in homogeneities will be effectively segmental

The experimental results have shown its promising leads to comparison with the present strategies. However, in case the mustache effect around the lip region becomes noticeable, the projected approach, similarly because the other existing ones, needs an image pre-processing to sight the region of mustache and mask it out[8].

The Lirong Wang and Xiaoli Wang suggest a new approach to perform the matter of real-time lip detection. The planned technique combines primitive Haar-Like feature and variance 
value to construct a new feature, supposed Variance based Haar-Like feature. They used SVM for coaching and classification, combining kalman filter will realize lip real time detection and chase. Experiments showed that face and lip detection system victimization Variance based Haar-Like feature and SVM can be rather more economical than face and detection system by victimization primitive Haar-Like features [9].

As per the Stefan M. Karlsson and Josef Bigun they recommended application of lip primarily based articulatory events detection has been shown to perform well on the XM2VTS info for the cases of mouth opening and closing events, as outlined by the first and second order time derivative of outer lip contour space. Conjointly the realm itself will be estimated, but with less accuracy [10]

Sasikumar Gurumurthy and B.K.Tripathy suggests the best way of automatic, strong and correct lip segmentation method. Its high flexibility enables very correct and realistic results. It makes this method very appropriate for applications that need a high level of preciseness such as lip reading. They introduced a replacement biometric identification system based on lip shape biomeasures, a field in which very little analysis has being done. This is considered as good result and encourage for its use combined with other bioscience systems [11].

According to Maycel Isaac Faraj and Josef Bigun describes a replacement motion based mostly feature extraction technique for speaker recognition victimization orientation estimation in second manifolds. The motion is calculable by computing the parts of the structure tensor from which traditional flows square measure extracted. The associate implementation based on joint lip movements and speech is presented together with experiments which confirm the speculation, exhibiting recognition [12].

E.S.Selvakumar and S.Shanmuga Priya they contribute to Automatic Speech Recognition (ASR) is an essential component in many Human-Computer Interaction systems. A variety of applications in the field of ASR have reached high performance levels but only for condition-controlled environments. In this work, they reduce the noise in the video lectures using bi-modal feature extraction. Audio signal features need to be enhanced with additional sources of complementary information to overcome problems due to large amounts of acoustic noise [13].

Hiroaki Kawashima, Yu Horii proposed a unique speech estimation methodology that directly estimates feature sequences of clean speech from ascertained mouth movements. Candidate generation is complete by exploitation hybrid selfpropelled systems (HDSs) and a temporal arrangement structure model between the HDSs. whereas the evaluation given during this paper is limited, it shows that the proposed methodology can estimate clean speech with much higher precision under non-stationary noise environments as compared to the strategies that use solely audio knowledge [14].

As per the Patrice Delmas As a result a fast and sturdy liptracking formula is bestowed here. First, comers and vertical extreme of the lips are extracted using brightness level, Hue and gradient info on areas and contours. Next, active contours are wont to extract lip contours for the current image. Then a variant of the Kanade-Lucas options point tracking technique permits a precise and fast format for the following image [15].

\section{PROBLEM FORMULATION}

The text based password authentication is the best method for preventing unauthorization of data accessibility form the un authorized person or intruders which are not the part of the system. But every system or a method has its own limitations such as the text based security can be easily broken by a simple brute force attack. So we have to improve this technique by finding some more way of security provider such as image based or motion based which are not easily recognized by others.

\section{SUGGESTED SOLUTION AND METHODOLOGY}

We are going to support password management system for the traditional text based security through image based or motion based recognizer. We can do this using either from a video file or from live streaming. The key idea is that we focus on the lip movement for a threshold value. By knowing the feature representation of outer lip contour and inner mouth features we will able to create a password.

Towards the Facial expression analysis based security system the Lips will play a major role in feature extraction for developing a biometric genuine system for user authentication. Thus the first inspiration of our work was to discover the behavioral feature of face recognition by using lip motion. We believe that the verbal communication blueprint is a unique behavioral attribute of human being that is acquire over time, which will be used as a biometric identifier to words password authentication for the users in upcoming scenario.

\subsection{Approach and Methodology}

As a contribution we are proposing a Lip based security system for this we need to use the combination of several independent methods like facial expression analysis, talk analysis and text analysis by using these things we will be provide a new authentication idea for the world towards data security or system authentication.

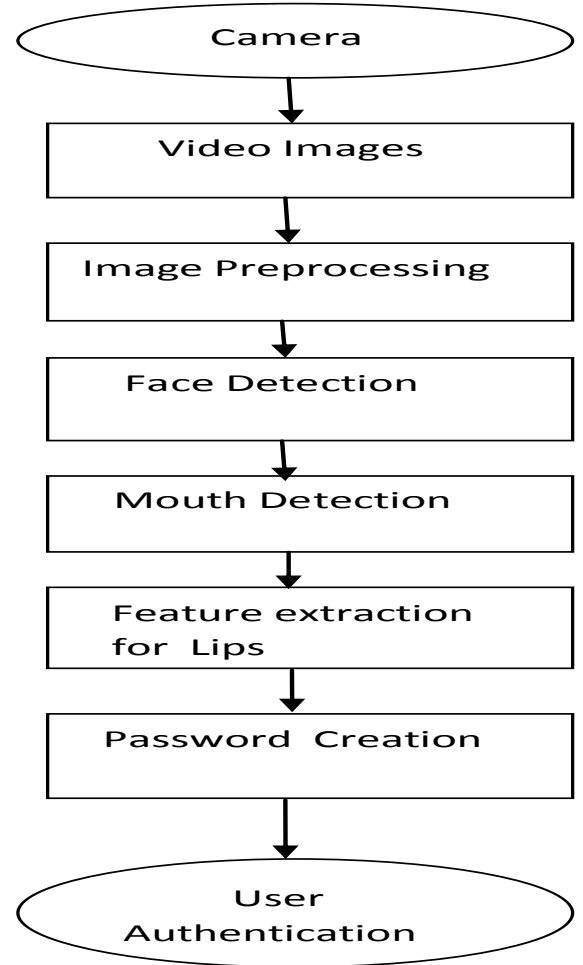

Fig 1: Proposed architecture for lip based user Authentication

We tend to are progressing to produce lip based password. We can do that using each from a video file or from live streaming. 
The main factor is that a camera should specialize in the lip. It should track the lip movement. The key plan is that we tend to are progressing to take a threshold value, using that specific value we tend to are progressing to check whether the password is correct or not. We will track the lip movement, if the movement is on the far side threshold then incorrect password otherwise correct.

\subsection{Proposed Algorithm}

Well here are the steps we would like to do to form lip based password creation based on motion of lips

Step 1.Take video of lip movement or record using digital camera.

Step 2.Now use a detection algorithmic program by applying the motion detection functions.

Step 3. Check for the threshold value by using the blocks. We will calculate the no of frames between one lip touching frame to future one in over case the calculated frame no will be the threshold value.

Step 4. So once the threshold is calculated check for the results.

Step 5. Now easy calculation if detected result is on the far side threshold then rejected otherwise accepted.

\subsection{Expected Outcomes}

To applying and implementing suggested approach, expect following conclusions.

- To retain reliability on system by proposing the solution to words security.

- Client ensures about data storage in safe manner and unauthorized access.

- To protect data from different attacks at client end.

- Might be became innovative approach to words image processing.

- Authentication can be achieved in a secure manner by motion of lips.

\section{CONCLUSION}

In this paper, we proposed a detailed method and approach for the Facial Expression Analysis of Feature Extraction Based on Lip Moment for User Authentication. Towards the Facial expression analysis based security system the Lips will play a major role in feature extraction for developing a biometric genuine system for user authentication. Thus the first inspiration of our work was to discover the behavioural feature of face recognition by using lip motion. We believe that the verbal communication blueprint is a unique behavioral attribute of human being that is acquire over time, which will be used as a biometric identifier to words password authentication for the users in upcoming scenario.

\section{ACKNOWLEDGMENTS}

This research work is self financed but recommended from the institute so as to improve the security solutions for authentication. Thus, the authors thank the anonymous reviewers for their valuable comments, which strengthened the paper. The authors also wish to acknowledge SVITS administration for their support \& motivation during this research. They also like to give thanks to Dr.Dinesh Chandra Jain who had guided me throughout this research and being held always for discussion regarding the security and polices \& for producing the approach adapted for this paper.

\section{REFERENCES}

[1] Sasirekha N and Hemalatha M, "An Enhanced Code Encryption Approach with HNT Transformations for Software Security", International Journal of Computer Applications (0975 - 8887) Volume 53- No.10, September 2012.

[2] Salah Werda, Walid Mahdi and Abdelmajid Ben Hamadou," Lip Localization and Viseme Classification for Visual Speech Recognition", International Journal of Computing \& Information Sciences, page no 67-75 Vol.5, No.1, April 2007, On-Line.

[3] Chao Sui, Mohammed Bennamoun, Roberto Togneri and Serajul Haque," A Lip Extraction Algorithm Using Region-based ACM With Automatic Contour Initialization", in IEEE 2012.

[4] Bouchra Abboud and G'erard Chollet," Appearance based lip tracking and cloning on speaking faces",in Proceedings of the 4th International Symposium on Image and Signal Processing and Analysis (2005).

[5] P. Vijaykumar, Aman Kumar and Sidharth Bhatia," Latest Trends, Applications and Innovations in Motion Estimation Research",in International Journal of Scientific \& Engineering Research Volume 2, Issue 7, July-2011.

[6] Eng-Jon Ong and Richard Bowden,” Robust Lip-Tracking using Rigid Flocks of Selected Linear Predictors",in IEEE 2008 .

[7] Usman Saeed," Person Identification using Behavioral Features from Lip Motion",In IEEE.

[8] Yiu-ming Cheung, Xin Liu , Xinge You,"A local region based approach to lip tracking" in iberian conference on pattern recognition and image analysis 2012, Elsevier.

[9] Lirong Wang, Xiaoli Wang and Jing Xu," Lip Detection and Tracking Using Variance Based Haar-like Features and Kalman filter" in Fifth International Conference on Frontier of Computer Science and Technology 2010.

[10] Stefan M. Karlsson and Josef Bigun,” Lip-motion events analysis and lip segmentation using optical flow" in IEEE 2012.

[11] Sasikumar Gurumurthy and B.K.Tripathy," Design and Implementation of Face Recognition System in Matlab Using the Features of Lips" I.J. Intelligent Systems and Applications, Published Online July 2012 in MECS.

[12] Maycel Isaac Faraj and Josef Bigun," Person Verification by Lip-Motion" in proceedings of Conference on Computer Vision and Pattern Recognition Workshop (CVPRW'06) IEEE 2006.

[13] E.S.Selvakumar and S.Shanmuga Priya," Noise Suppression in Tele-Lectures using Bi-Modal Feature Extraction" published in International Journal of Computer Science and Network (IJCSN), Vol 2, Issue 2, April 2013.

[14] Hiroaki Kawashima, Yu Horii and Takashi Matsuyama," Speech Estimation in Non-Stationary Noise Environments Using Timing Structures between Mouth Movements and Sound Signals" in IEEE.

[15] Patrice Delmas, Nicolas Eveno and Marc Lievin," Towards Robust Lip Tracking" published in ICPR, IEEE2002. 